\title{
ESTUDO PARAMÉTRICO DA COGASEIFICAÇÃO DE CARVÃO MINERAL E CASCA DE ARROZ PARA A PRODUÇÃO DE SYNGAS ENRIQUECIDO EM HIDROGÊNIO.
}

\author{
S. R., PETERS ${ }^{1}$, R. ZOTTIS ${ }^{1}$, A.R.C. MUNIZ1 ${ }^{1}$, R. RODRIGUES ${ }^{1}$ \\ ${ }^{1}$ Universidade Federal do Pampa, Curso de Engenharia Química \\ E-mail para contato: steffany.rpeters@gmail.com
}

\begin{abstract}
RESUMO - A cogaseificação é considerada uma alternativa sustentável, que possibilita a conversão termoquímica de materiais carbonosos, gerando o syngas. Este gás de síntese pode ser empregado na cogeração de energia e em processos industriais como, a produção de hidrogênio e a sínteses de produtos químicos. Neste trabalho simulou-se no software UniSim ${ }^{\circledR}$ Design a cogaseificação de carvão mineral (CM) e casca de arroz (CA) afim de obter syngas para a produção de um syngas enriquecido em hidrogênio. Foram avaliados os seguintes parâmetros: razão de alimentação de carvão mineral e casca de arroz; e a razão entre os combustíveis da mistura e os agentes de gaseificação. Verificou-se que o aumento do teor de CA no combustível, acarreta uma baixa produção de $\mathrm{H}_{2}$ do syngas. Observou-se que a temperatura, relação Ar/Combustível e relação $\mathrm{S} / \mathrm{F}$ são parâmetros de forte influência na composição do syngas. Conclui-se que a alimentação de $77 \%$ de CM e $23 \%$ de CA produz altos teores de $\mathrm{H}_{2}(19$ a $28 \%$ ) e baixos teores de $\mathrm{CO}$ (4 a 8,5 \%), condição mais promissora para a operação do gaseificador.
\end{abstract}

\section{INTRODUÇÃO}

O Rio Grande do Sul possui a maior jazida de carvão mineral do país, localizada no município de Candiota (ANEL, 2002), além de ser o Estado que mais produz arroz (IBGE, 2016). Consequentemente é o maior gerador de casca de arroz, resíduo proveniente do beneficiamento do grão. A gaseificação pode ser considerada uma alternativa viável para o processamento destes dois combustíveis. Esta tecnologia possibilita o uso de dois materiais carbonosos (biomassa e combustível fóssil), denominada de "cogaseificação", o qual gera um gás (syngas) que pode ser utilizado na produção de hidrogênio e sínteses de produtos da indústria química.

Com o auxílio da modelagem, simulação e otimização de processos industriais, grandes progressos tecnológicos foram alcançados, pois almejam a melhoria de processos existentes ou, criações de novas plantas industriais.

Neste contexto, simulou-se no software UniSim ${ }^{\circledR}$ Design (Honeywell, Inc) a cogaseificação de carvão mineral (CM) e casca de arroz (CA) afim de obter syngas rico em hidrogênio. Foram avaliados os seguintes parâmetros: razão de alimentação de carvão mineral e casca de arroz; e a razão entre os combustíveis da mistura e os agentes de gaseificação.

\section{METODOLOGIA}


A simulação do processo de cogaseificação foi realizada em estado estacionário no software UniSim ${ }^{\circledR}$ Design, versão 440.

A composição das correntes de carvão mineral $(\mathrm{CM})$ e a casca de arroz (CA) foram especificadas pelas análises imediata e elementar conforme quantificado por BEHAINE, et al (2000) e MORAIS et al (2006), como mostra a Tabela 1.

Tabela 1-Análise imediata e elementar do carvão mineral e da casca de arroz.

\begin{tabular}{cccc}
\hline & & $\mathbf{C M}^{\mathbf{1}}$ & $\mathbf{C A}^{\mathbf{2}}$ \\
\hline & Umidade Total & 10,5 & 10,90 \\
Análise & Material Volátil & 24,0 & 81,60 \\
Imediata (\%), bu) & Carbono Fixo & 22,6 & 8,10 \\
& Teor de cinzas & 41,19 & 10,30 \\
& PCS (kJ/kg) & 11880 & 15330 \\
\hline & Carbono & 33,61 & 40,1 \\
Análise & Hidrogênio & 3,38 & 4,7 \\
Elementar $(\% \mathbf{p})$ & Oxigênio & 7,28 & 38,3 \\
& Nitrogênio & 0,46 & 0,4 \\
& Enxofre & 1,85 & 0,2 \\
\hline
\end{tabular}

Fonte: adaptado de (1) BEHAINE, et al (2000) e (2) MORAIS et al (2006)

Utilizou-se ar e vapor de água como agentes de gaseificação. As correntes de ar e vapor de água foram especificadas a pressão atmosférica a $25^{\circ} \mathrm{C}$ e $100^{\circ} \mathrm{C}$, respectivamente.

Considerou-se na simulação um gaseificador de leito fluidizado pois é um dos reatores mais utilizados na indústria em processos de CM e biomassa segundo (BRAR et al, 2012). As especificações do gaseificador foram baseadas em um modelo comercial leito fluidizado modelo High Temperature Winkler (HTW ${ }^{\mathrm{TM}}$ ) do fabricante ThyssenKrupp, que opera com 1,6 a 30 t/h de combustível, produzindo de 3000 a $34000 \mathrm{Nm}^{3} /$ dia de gás (MELO, 2016), no caso de CM. Restringiu-se a operação do reator de 700 a $900{ }^{\circ} \mathrm{C}$, faixa relatada pela literatura para gaseificadores de leito fluidizado. Fixou-se a vazão total da mistura de combustíveis em 30 t/h. Variou-se a razão entre CM e biomassa e, concomitantemente, a vazão de ar e a vazão de vapor de água ( 0 a 1,2 e 0,43 a 1,0, respectivamente). Nestas simulações foram avaliadas a composição e temperatura do syngas.

Para a simulação da cogaseificação, considerou-se que a mistura entre o CM e a CA atingiu o equilíbrio químico, deste modo, a simulação ocorreu em um reator termodinâmico (reator de Gibbs) não-isotérmico. Este reator não necessita do conhecimento da estequiometria e cinética das reações químicas envolvidas no processo de cogaseificação, mas, somente das espécies que participarão das reações de conversão termoquímica.

O esquema da simulação da cogaseificação no simulador UniSim® Design está apresentado na Figura 1. 
Figura 1- Esquema da simulação da cogaseificação a pressão atmosfera.

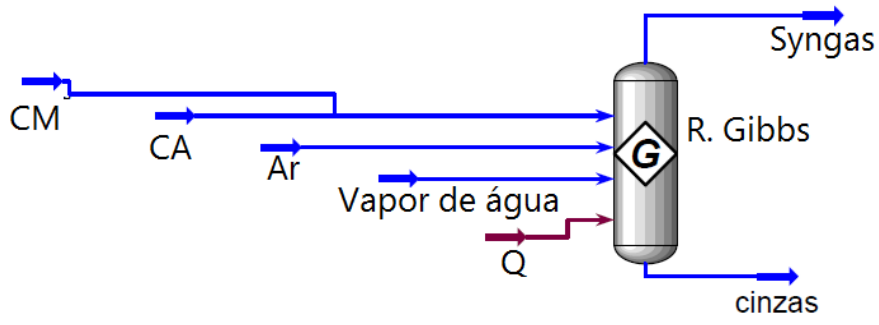

Fonte: Autor (2016)

Afim de se obter resultados mais próximos a realidade a corrente de energia (Q) foi proposta. A estimativa desta, envolveu uma correção na energia fornecida pelos agentes gaseificantes e combustíveis utilizando a entalpia de formação do CM e da CA. Também se considerou $10 \%$ de perdas de energia total para o meio ambiente através das paredes do reator.

\section{RESULTADOS E DISCUSSÕES}

Foi analisada a influência da mistura entre o CM e a CA na composição do syngas. Na Figura 2, constatou-se uma influência relevante do teor de CA na composição do gás. Comparando-se a simulação da mistura entre CM e CA com o trabalho experimental realizado por ANDRÉ (2007), que cogaseificou o bagaço do caroço de azeitona com CM à uma temperatura de $845^{\circ} \mathrm{C}$.

Figura 2 - Composição do syngas em relação ao teor de casca de arroz a $845^{\circ} \mathrm{C}$

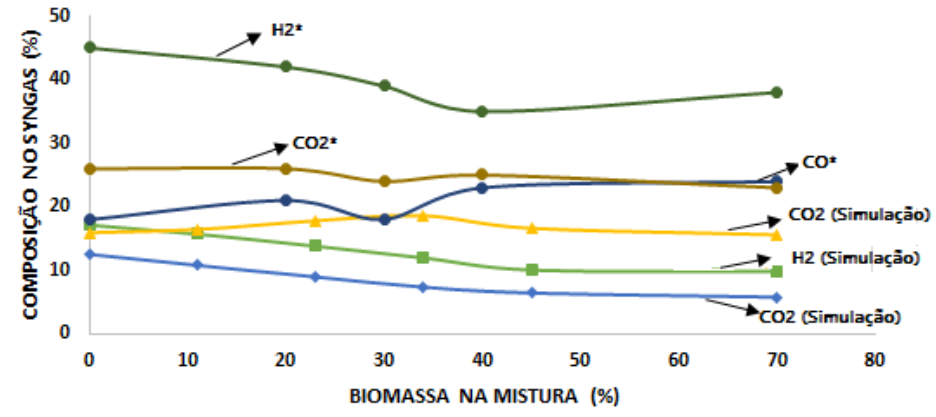

Fonte: *ANDRÉ (2007)

Verificou-se que teores de CA superior a 35\% na mistura do combustível, acarretou em um gás com menor composição de $\mathrm{H}_{2}$ e $\mathrm{CO}$, enquanto, a composição de $\mathrm{CO}_{2}$ apresentou pequenas variações. ANDRÉ (2007) também observou o mesmo comportamento em relação à composição do $\mathrm{H}_{2}$, porém, com maiores quantidades de bagaço do caroço de azeitona na mistura, obteve maior formação de CO no gás.

Observou-se o comportamento da temperatura do gaseificador e composição de $\mathrm{H}_{2}$ no syngas proveniente da cogaseificação de uma mistura de $15 \%$ de CA, como mostra a Figura 3. Comparou-se a simulação com o trabalho experimental de CHEJNE et al (2009), que gaseificou $\mathrm{CM}$ e $15 \%$ de $\mathrm{CA}$ em um leito fluidizado. 
Figura 3 - Composição do $\mathrm{H}_{2}$ no syngas em função da temperatura

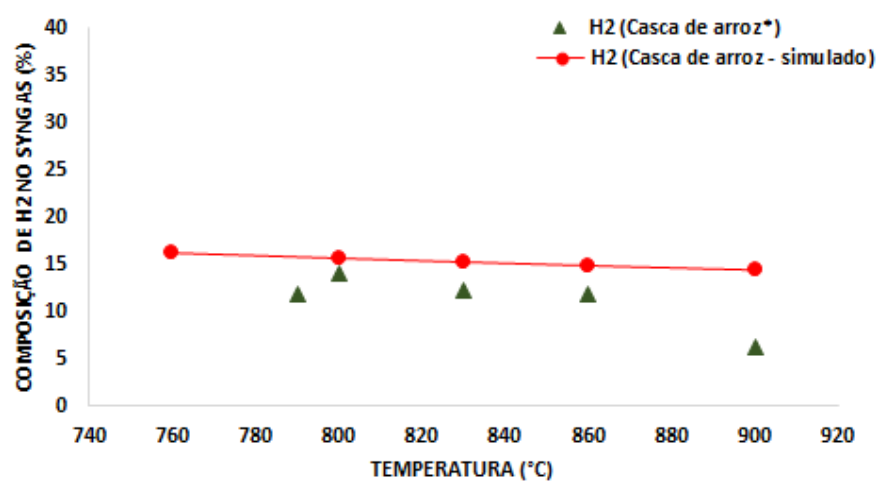

Fonte: *CHEJNE et al (2009)

Verificou-se resultados muito próximos para os teores da CA experimental e simulada, na faixa de 800 a $860{ }^{\circ} \mathrm{C}$. Para a CA simulada, o maior teor de $\mathrm{H}_{2}$ foi a $760{ }^{\circ} \mathrm{C}$. Enquanto, para a CA da literatura a $800{ }^{\circ} \mathrm{C}$. Observa-se uma diminuição no teor de $\mathrm{H}_{2} \mathrm{com}$ o aumento da temperatura do processo de cogaseificação. Este comportamento pode ter ocorrido devido à alta quantidade de vapor de água injetado, para assim, obter a faixa de temperatura ideal do reator de leito fluidizado. El-Rub et al (2004) mostra que utilizando uma fração alta de vapor de água, ocorre elevação do teor de $\mathrm{H}_{2}$ em temperatura baixas $\left(600{ }^{\circ} \mathrm{C}\right)$. Segundo RADWAN (2012) a temperatura do gaseificador afeta a velocidade de reação e a composição do produto, resultando um maior teor de $\mathrm{H}_{2}$. Mas, segundo $\mathrm{ABCM}$ a conversão de $\mathrm{CM}$ diminui com o aumento da temperatura (acima de $900^{\circ} \mathrm{C}$ ) que, por consequência, promove a fusão da escória (cinza). Então, para esse trabalho torna-se positivo que as maiores frações de $\mathrm{H}_{2}$ encontrarem-se nas faixas de temperatura de 700 a $900^{\circ} \mathrm{C}$.

A partir da análise realizada na Figura 2, foram selecionadas as duas misturas entre o $\mathrm{CM}$ e a CA que obtiveram a maior fração de $\mathrm{H}_{2}$ no syngas. Tais misturas foram: Mistura 1 (M1), 89\% CM e 11\% CA; Mistura 2 (M2), 77\% CM e 23\% CA. Deste modo, realizou-se uma análise em relação aos parâmetros de entrada (vazão de ar e de vapor de água), as composições de $\mathrm{H}_{2}$ e $\mathrm{CO}$ no syngas e temperatura, para assim, determinar a mistura ideal para o processo de produção do hidrogênio. A Figura 4 apresenta a variação do $\mathrm{H}_{2}$ e do $\mathrm{CO}$ em relação à vazão de ar e vapor de água na entrada do reator.

Figura 4 - Composição do syngas em relação a vazão de ar e vapor.
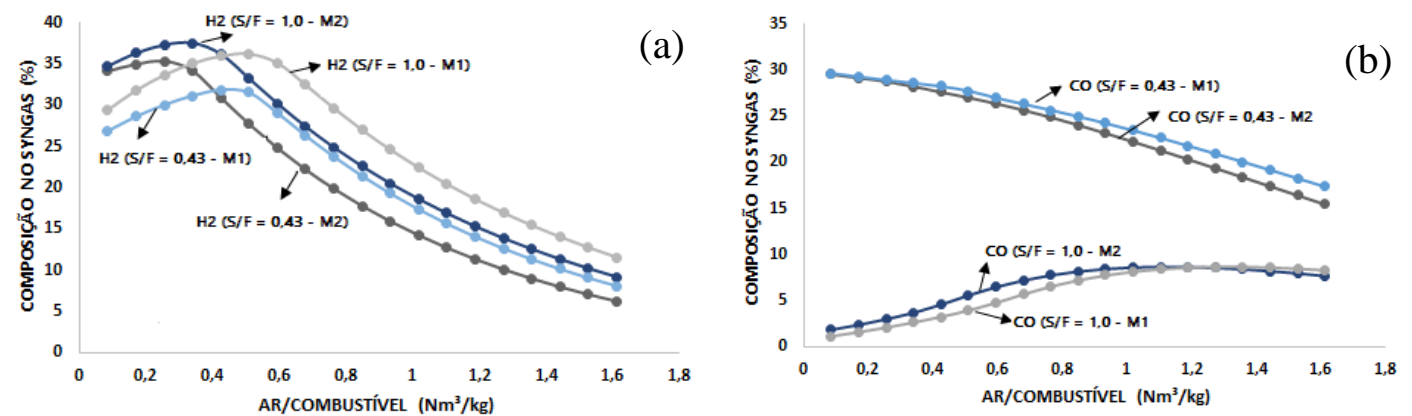

Mistura 1 (M1) - 89\% CM e 11\% CA; Mistura 2 (M2) - 77\% CM e 23\% CA; S/F - Vazão de Vapor/Combustível 
$\mathrm{Na}$ Figura 4(a), nota-se que as maiores composições de $\mathrm{H}_{2}$ corresponde as menores faixas de Ar/Combustível (M1: entre 0,3 a 0,6 e M2: entre 0 a 0,42). Porém, estas localizamse em regiões de temperaturas inferiores a $710{ }^{\circ} \mathrm{C}$. Por isso, foram consideradas para avaliação deste trabalho, as faixas de Ar/Combustível de (M1: 0,7 a 1,2 e M2: entre 0,6 a 1,0) que estão dentro da faixa de temperatura de operação do gaseificador de leito fluidizado.

Na Figura 4(b), aumentando a vazão de ar, notou-se que a relação S/F igual a 0,43 a composição do $\mathrm{CO}$ diminuiu. Porém, quando se utilizou a relação $\mathrm{S} / \mathrm{F}$ igual a 1,0, ocorreu um aumento da composição de $\mathrm{CO}$. Mas, mesmo ocorrendo esse aumento de $\mathrm{CO}$, a fração deste componente para as duas misturas permaneceu abaixo de $10 \%$ nas faixas de temperatura de operação do gaseificador de leito fluidizado.

Estimou-se que a mistura M2 foi mais promissora ao processo de produção de hidrogênio. Obteve-se altos teores de $\mathrm{H}_{2}(19$ a $28 \%)$ e baixos teores de $\mathrm{CO}(4,0$ a 8,5 \%) utilizando uma relação S/F igual a 1,0 e um menor teor de CM na mistura do combustível.

\section{CONCLUSÃO}

A partir das simulações da cogaseificação foi possível verificar que o aumento do teor de CA no combustível, acarreta uma baixa produção de $\mathrm{H}_{2}$ do syngas. Os parâmetros temperatura, razão Ar/Combustível e relação $\mathrm{S} / \mathrm{F}$ apresentam grande influência na composição do syngas.

A mistura de $77 \%$ de CM e $23 \%$ de CA mostrou-se a mais promissora para a obtenção de um syngas enriquecido em hidrogênio, pois possui altos teores de $\mathrm{H}_{2}$ (19 a $28 \%$ ) e baixos teores de $\mathrm{CO}(4$ a $8,5 \%)$.

\section{NOMENCLATURA}

CA - Casca de Arroz

CM - Carvão Mineral

$\mathrm{Q}(\mathrm{kJ} / \mathrm{h})$ - Corrente de energia

$\mathrm{S} / \mathrm{F}\left(\mathrm{Nm}^{3} / \mathrm{kg}\right)$ - Vazão vapor/combustível

\section{REFERENCIAS}

ABCM - Associação Brasileira de Carvão Mineral. Disponível em: <http://www.carvaomineral.com.br>. Acesso em: 28 de março de 2017.

ABU EL-RUB EA BRAMER Z.; BREM, G. Review of Catalysts for Tar Elimination in Biomass Gasification Processes. Ind Eng Chem Res; 2004, 43:6911-9 
AGÊNCIA NACIONAL DE ENERGIA ELÉTRICA. Carvão mineral. Atlas de Energia Elétrica do Brasil. 2002. Disponível em: www.aneel.gov.br/arquivos/pdf/atlas_par3_cap9.pdf>. Acesso em: 26 de março, 2017.

ANDRÉ, R. M. N. Desenvolvimento do processo de co-gasificação de carvão com resíduos. Tese de Doutorado. Departamento de Ambiente e Ordenamento - Universidade de Aveiro. Aveiro, 2007.

BEHAINE, John J. R.; SÁNCHEZ, Caio G. Diminuição de H2S na gaseificação de carvão mineral mediante adição de dolomita em reator de leito fluidizado. CONGRESSO BRASILEIRO DE ENGENHARIA E CIÊNCIAS TÉRMICAS - ENCIT 2000. Porto Alegre. Anais eletrônicos. Porto Alegre. ENCIT, 2000.

BRAR, J. S.; SINGH, K.; WANG, J; KUMAR, S. Cogasification of coal and biomass: A review. Int. J. Forest. Res., 363058, p. 1-10 (2012).

CHEJNE, F; Vélez, J. F; VALDÉS, C. F.; EMERY, E. J.; LONDOÑO, C. A. Co-gasification of Colombian coal and biomass in fluidized bed: An experimental study. Journal Elsevier - Fuel 88 (2009) $424-430$.

IBGE - INSTITUTO BRASILEIRO DE GEOGRAFIA E ESTATÍSTICA. Informações das culturas permanentes e temporárias do Brasil: IBGE, 2016.

MELLO, L. Thyssenkrupp Industrial Solutions' PRENFLO ${ }^{\circledR}$ and HTWTM Gasification Technologies. World Clean Coal Conference - Gasification Technology. Rio de Janeiro - June 22-23, 2016.

MORAIS, M; SEYE, O; FREITAS, K. T; RODRIGUES, M; SANTOS, E. C. S; SOUZA, R. Obtenção de briquetes de carvão vegetal de casca de arroz utilizando baixa pressão de compactação. Manaus, 2006. 